International Journal of Pure and Applied Mathematics

Volume 84 No. 3 2013, 163-174

ISSN: 1311-8080 (printed version); ISSN: 1314-3395 (on-line version)

url: http://www.ijpam.eu

doi: http://dx.doi.org/10.12732/ijpam.v84i3.3

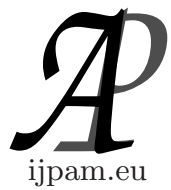

\title{
STOCHASTIC MODEL OF BRITTLE CRACK GROWTH UNDER CYCLIC LOAD
}

\author{
Boris Kunin \\ University of Alabama in Huntsville \\ Huntsville, AL 35899, USA
}

\begin{abstract}
An existing model of slow crack growth in brittle composites is extended to the case of cyclic loading. The mode of crack growth being modeled consists of a sequence of jumps of random magnitude with random arrest durations between the jumps. Both the jumps and the arrest durations are governed by their respective non-homogeneous Poisson processes, one in space coordinate, the other one in the number of cycles $N$. A PDE is derived for the transition probability of the random process that represents crack tip location as a function of $N$. A Paris Law type relation is derived. A numerical illustration is considered.
\end{abstract}

AMS Subject Classification: 74R10, 60K35

Key Words: brittle fracture, stochastic Crack growth, Paris law

\section{Introduction}

This paper is devoted to a model of quasi-static random crack growth. It further develops ideas examined in [1]. Crack growth is modeled as a sequence of microscopic jumps of random length, each one followed by an arrest of random duration. An alternative approach to random crack formation can be found in [2]. We attempt to come closer to modeling Paris Law. The latter is a successful summary of abundance of experimental results in fatigue crack growth. Paris Law claims that, under cyclic loading, the crack speed is proportional to a

Received: October 24, 2012

(c) 2013 Academic Publications, Ltd. url: www.acadpubl.eu 
power of the amplitude of the stress intensity factor over a wide range of the values of the latter (see Section 4.2).

The model described in [1] was shown to produce a crack-speed-vs.-stressintensity-factor relationship that qualitatively resembled those observed experimentally. In order to aspire to be a "micromodel of the Paris Law", the model had to be modified.

Firstly, in [1], the average crack speed represented the rate of change of the crack length with time. One of the observations made by Paris and Erdogan for cyclic loading is that the proper independent variable is the number of cycles, not time. Thus, the crack speed to be considered is the rate of change of the crack length with respect to the number of cycles.

Secondly, the load in [1] was assumed static, so the crack-speed-vs.-stressintensity-factor relation there was only an analog of the Paris Law at best.

In this paper, we assume load to be cyclic and modify the assumptions of the model in order for the number of cycles to become the time-like variable. We then derive an equation for the time-dependent probability distribution of crack tip locations (Sections 2, 3).

In Section 4, we show the results of computations for a tapered compact tension specimen. Those include evolution of crack length distribution and the relation between the average crack speed and the amplitude of the stress intensity factor (Paris Law type relationship).

\section{Modeling Crack Jumps and Crack Arrest Durations}

We consider crack propagation along the $x$-axis (see Fig. 1). "Crack jump" stands for an event during which a previously arrested crack instantaneously advances and is then arrested, with its tip at a new location. Material in front of the crack is viewed as a sequence of randomly distributed obstacles. Once initiated, the crack jumps through all of the obstacles it can overcome and is arrested at the first 'insurmountable obstacle' (see below). Thus, the length of each jump is random. What happens after the arrest is discussed in Section 3.

Following [1] we introduce the probability that a crack with its tip at $x$ will overcome all of the obstacles between $x$ and an arbitrary $X \geq x$ (and, therefore, the crack arrest would occur to the right of $X$ ). We refer to this function of $X$ and $x$ as Crack Propagator (see the definition below).

Consider a solid with a straight crack (Fig. 1). We will refer to the $x$ coordinate of the crack tip as crack depth. Assume that the applied loading 

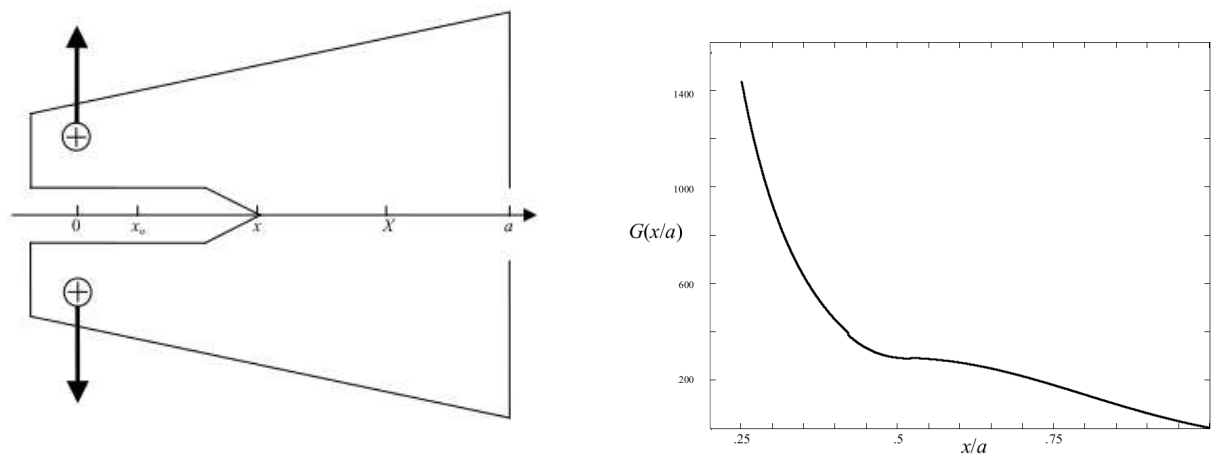

Figure 1: An example of specimen-loading configuration to be considered and the corresponding typical graph of $G(x / a)$ (see Section 4).

is symmetric relative to the crack line to justify rectilinear crack growth along the $x$-axis. We assume that, in the course of the instantaneous phase, the crack, its tip currently at $x$, will advance by another $d x$ if the elastic energy release resulting from crack advancing from $x$ to $x+d x$ would be greater than the energy required for breaking the material between $x$ and $x+d x$. The (linear) "elastic energy release" is $G(x) d x$, where $G(x)$ is considered a known deterministic function, the so-called energy release rate. The "energy required for breaking" is taken to be $2 \gamma(x) d x$, where $\gamma(x)$ is the value of a random field $\gamma$ at $x$. (When $\gamma$ is a constant they refer to it as specific fracture energy.) Thus, "no arrest at $x$ " means $2 \gamma(x) \leq G(x)$.

Beginning with Section 3, we will assume that the load and, consequently, $G$ may vary with time. This will not invalidate what immediately follows, since each jump is instantaneous, and so $G$ as a function of $x$ is fixed during the jump.

We make the following assumption about the random field $\gamma:^{1}$

(a) $\gamma$ is homogeneous in $x$,

(b) values of $\gamma$ at every point are governed by a Weibull distribution,

$$
F(\gamma)= \begin{cases}1-\exp \left(-\left[\Gamma\left(1+\frac{1}{\alpha}\right) \frac{\gamma-\gamma_{\min }}{\gamma^{*}-\gamma_{\min }}\right]^{\alpha}\right) & \text { if } \gamma \geq \gamma_{\min } \\ 0 & \text { if } \gamma<\gamma_{\min }\end{cases}
$$

\footnotetext{
${ }^{1}$ One can find a discussion of the assumed properties of $\gamma$ and consequences, 'in physical terms', in [4], [5].
} 
Here the parameters $\gamma^{*}$ and $\gamma_{\min }$ are the average and minimal values of $\gamma$, respectively, and $\alpha>0$ is known as a 'shape parameter' - they characterize the scatter of material's strength on a microscale; $\Gamma(\cdot)$ is the $\Gamma$-function,

(c) properties of $\gamma$ on disjoint intervals are independent, and

(d) for any coordinate $x \geq x_{0}$ and for any number $g \geq \gamma_{\min }$, the probability of $\gamma$ exceeding $g$ somewhere between $x$ and $x+d x$ is proportional to $d x$, specifically,

$$
\operatorname{Prob}\{\gamma>g \text { somewhere on }(x, x+d x]\}=(1-F(g)) \frac{d x}{r},
$$

where $r>0$ is a parameter. (When the solid under consideration is finite, one assumes that $r$ is small in comparison to the solid's size. $)^{2}$

Therefore, the probability of the crack being arrested on $(x, x+d x]$ is

$$
\operatorname{Prob}\{2 \gamma>G(x) \text { somewhere on }(x, x+d x]\}=\frac{U(x)}{r} d x,
$$

where $U(x)=1-F\left(\frac{G(x)}{2}\right)$.

For two arbitrary points $x$ and $X, x \leq X$, on the $x$-axis let us define crack propagator $(\mathrm{CP}),[X \mid x]$, as the probability that the crack can advance instantaneously to at least $X$ conditional on that either the crack has already passed through $x$ or the initiation at $x$ has occurred. (One can formally define $[X \mid x]=0$ for $x>X$, which would express the assumption that cracks do not cure.) The definition is a one-dimensional case of the notion introduced in [3]. Notice that if $x$ were called "time", then the above assumptions would mean that the random event of crack arrest is governed by a non-homogeneous Poisson process with "time"-dependent intensity $U(x) / r$. The absence of crack arrest between $x$ and $X$ (conditional, as described above), corresponds, in the Poisson process terminology, to having "zero number of events in "time" interval $(x, X]$ ". Its probability is therefore expressed by a well known formula:

$$
[X \mid x]=\exp \left\{-\int_{x}^{X} \frac{U(\xi)}{r} d \xi\right\} .
$$

Thus, the explicit expression for CP becomes (for $x \leq X$ )

$$
[X \mid x]=\exp \left\{-\int_{x}^{X} \exp \left(-\left[\Gamma\left(1+\frac{1}{\alpha}\right) \frac{\frac{G(\xi)}{2}-\gamma_{\min }}{\gamma^{*}-\gamma_{\min }}\right]^{\alpha}\right) \frac{d \xi}{r}\right\} .
$$

\footnotetext{
${ }^{2}$ For examples of experimental evaluation of the parameters $\gamma^{*}, \gamma_{\min }, \alpha$, and $r$ of the $\gamma$-field, see [6], [7].
} 
Evidently, $[X \mid x]$ is determined by the energy release function $G(x)$. If the latter varies with the number of cycles $N$, then so does $[X \mid x]$. In the latter case, $[X \mid x]$ depends on $N$.

The following useful property of $\mathrm{CP}$ is obvious from its definition and the independence of properties of the field $\gamma$ on disjoint intervals (as well, as from Eq. (3)): for any $x \leq x^{\prime} \leq X$,

$$
\left[X \mid x^{\prime}\right]\left[x^{\prime} \mid x\right]=[X \mid x] .
$$

If the $\mathrm{CP}$ varies with $N$, then all three expressions in Eq. (5) refer to the same value of $N$, i.e. to the same 'instant'.

In this paper, we deal with 'stable' specimen-loading configurations exemplified by Fig. 1. In [1], unstable configurations were considered. We call a specimen-loading configuration stable, if, upon initiation, a crack of any length will surely be arrested (as opposed to there being a non-zero probability of the crack reaching the specimens right edge and thus breaking the specimen in two). ${ }^{3}$ Formally, if $a$ denotes the $x$-coordinate of the right edge of the specimen (we include the possibility $a=\infty$ ), then we are assuming $[a \mid x]=0$ for all $x \geq x_{0}$. This is guaranteed to happen if $G(x) \rightarrow 0$, as $x \rightarrow a$.

From now on, let us assume that the loading is cyclic. On the basis of empirical data, it has been recognized that, under cyclic loading, crack growth correlates well with the number of cycles. (Paris Law, see Section 4.2, is an illustration of this statement.) For this reason, the assumption about the duration of crack arrest that we make next involves cycles and not time.

Let $N$ denote the number of cycles that have elapsed since the loading started. Suppose that the crack is in the state of arrest, its tip at $x$. We make the following assumption about the likelihood of a crack jump between cycles $N$ and $N+d N$.

$$
\operatorname{Prob}\{\text { initiation from } x \text { during } d N\}=\lambda\left(\gamma^{*}, G(x, N)\right) \frac{d N}{\nu}
$$

Here $\nu$ (a characteristic "time scale" for the submicroscopic processes) is introduced explicitly to render the function $\lambda\left(\gamma^{*}, G\right)$ dimensionless. As was pointed out in [1], in the absence of other relevant dimensional constants, $\lambda\left(\gamma^{*}, G\right)$ has to be a function of $\gamma^{*} / G$. One choice of $\lambda$ is made in Section 4 .

Evidently, we have assumed that, for a crack arrested at $x$, its subsequent initiation is governed by a Poisson process, the number of cycles $N$ playing the role of time, with the variable intensity $\lambda\left(\gamma^{*}, G(x, N)\right) / \nu$, the intensity varying

\footnotetext{
${ }^{3}$ Stable configurations typically occur when a solid is loaded through prescribed displacements ("displacement controlled loading").
} 
with $N$ ( $x$ is fixed). For convenience, let us denote $\Lambda(x, N)=\lambda\left(\gamma^{*}, G(x, N)\right)$. Then the probability that initiation does not happen between cycles $n$ and $N \geq n$ is

$$
\begin{gathered}
\text { Prob } \left.\begin{array}{c}
\left\{\begin{array}{c|c}
\text { no crack initiation from } x \\
\text { between } n \text { and } N \geq n
\end{array}\right. \\
=\exp \left(-\int_{n}^{N} \frac{\Lambda\left(x, N^{\prime}\right)}{\nu} d N^{\prime}\right) \\
\text { at } x \text { prior to } n
\end{array}\right\}
\end{gathered}
$$

\section{Distribution of Crack Arrest Locations as a Function of Time}

Let $\xi(N)$ denote the rightmost position of the crack tip at a cycle $N$. The random process $\xi(N)$ has monotonically growing piecewise constant realizations. Also, notice that the statement $\xi(N)=x$ implies that, at $N$, the crack tip is at $x$ and the crack is in the state of arrest.

Denote by $P(x, N)$ the probability that, at a cycle $N$, the crack depth is less than or equal $x$, assuming that, at $N=0$, the crack tip was at $x_{0}$ in the state of arrest, $P(x, N)=\operatorname{Prob}\left\{\xi(N) \leq x \mid \xi(0)=x_{0}\right\}$.

The following derivation of a PDE for $P(x, N)$ parallels that in [1]. Under the present circumstances, each $\mathrm{CP}$ below depends on $N$, and we indicate this dependence explicitly, e.g. $[X \mid x ; N]$. Let $x \geq x_{0}$ and $N \geq 0$. Assuming that $\xi(N) \leq x$, there are two and only two mutually exclusive possibilities to have $\xi(N+d N) \leq x$ :

(a) $\xi(N)=x_{0}$ (in the state of arrest) and, during $[N, N+d N]$, it did not happen that crack initiation occurred and was followed by an instantaneous crack jump to a depth greater than $x$; the probability of this is

$$
P\left(x_{0}, N\right)\left(1-\frac{\Lambda\left(x_{0}, N\right) d N}{\nu}\left[x \mid x_{0} ; N\right]\right) ;
$$

(b) $\xi(N) \in\left(x^{\prime}, x^{\prime}+d x^{\prime}\right]$ for some $x^{\prime} \in\left(x_{0}, x\right]$ and, during $[N, N+d N]$, it did not happen that crack initiation occurred and was followed by an instantaneous crack jump to a depth greater than $x$; the probability of $\xi(N) \in\left(x^{\prime}, x^{\prime}+d x^{\prime}\right]$ is $P\left(x^{\prime}+d x^{\prime}, N\right)-P\left(x^{\prime}, N\right)=P_{x}\left(x^{\prime}, N\right) d x^{\prime},{ }^{4}$ the probability of crack initiation that is followed by an instantaneous crack advance beyond $x$ is $\frac{\Lambda\left(x^{\prime}, N\right) d N}{\nu}\left[x \mid x^{\prime} ; N\right]$; since, for non-intersecting intervals

\footnotetext{
${ }^{4}$ Subscripts stand for partial derivatives. Here, $P_{x} \equiv \frac{\partial P}{\partial x}$.
} 
$\left(x^{\prime}, x^{\prime}+d x^{\prime}\right]$, the events $\xi(N) \in\left(x^{\prime}, x^{\prime}+d x^{\prime}\right]$ are mutually exclusive, then the probability of the event under consideration is

$$
\int_{x_{0}}^{x} P_{x}\left(x^{\prime}, N\right)\left(1-\frac{\Lambda\left(x^{\prime}, N\right) d N}{\nu}\left[x \mid x^{\prime} ; N\right]\right) d x^{\prime} .
$$

Thus,

$$
\begin{aligned}
P(x, N+d N)= & P\left(x_{0}, N\right)\left(1-\frac{\Lambda\left(x_{0}, N\right) d N}{\nu}\left[x \mid x_{0} ; N\right]\right) \\
& +\int_{x_{0}}^{x} P_{x}\left(x^{\prime}, N\right)\left(1-\frac{\Lambda\left(x^{\prime}, N\right) d N}{\nu}\left[x \mid x^{\prime} ; N\right]\right) d x^{\prime} \\
=\quad & -P\left(x_{0}, N\right) \frac{\Lambda\left(x_{0}, N\right) d N}{\nu}\left[x \mid x_{0} ; N\right] \\
& +P\left(x_{0}, N\right)+\int_{x_{0}}^{x^{2}} P_{x}\left(x^{\prime}, t\right) d x^{\prime} \\
& -\int_{x_{0}}^{x} P_{x}\left(x^{\prime}, N\right) \frac{\Lambda\left(x^{\prime}, N\right) d N}{\nu}\left[x \mid x^{\prime} ; N\right] d x^{\prime} \\
= & -P\left(x_{0}, N\right) \frac{\Lambda\left(x_{0}, N\right) d N}{\nu}\left[x \mid x_{0} ; N\right]+P(x, N) \\
& -\int_{x_{0}}^{x} P_{x}\left(x^{\prime}, N\right) \frac{\Lambda\left(x^{\prime}, N\right) d N}{\nu}\left[x \mid x^{\prime} ; N\right] d x^{\prime} .
\end{aligned}
$$

From Eq. (5), we have $\left[x \mid x^{\prime} ; N\right]=\left[x \mid x_{0} ; N\right] /\left[x^{\prime} \mid x_{0} ; N\right]$. Substituting this into the integral above we get

$$
\begin{aligned}
P(x, N+d N)= & -P\left(x_{0}, N\right) \frac{\Lambda\left(x_{0}, N\right) d N}{\nu}\left[x \mid x_{0} ; N\right]+P(x, N) \\
& -\left[x \mid x_{0} ; N\right] \int_{x_{0}}^{x} \frac{P_{x}\left(x^{\prime}, N\right)}{\left[x^{\prime} \mid x_{0} ; N\right]} \frac{\Lambda\left(x^{\prime}, N\right) d N}{\nu} d x^{\prime}
\end{aligned}
$$

Move $P(x, N)$ to the left hand side, divide by $d N$, and factor out $\left[x \mid x_{0} ; N\right]$ to get

$$
P_{N}(x, N)=-\left[x \mid x_{0} ; N\right]\left(P\left(x_{0}, N\right) \frac{\Lambda\left(x_{0}, N\right)}{\nu}+\int_{x_{0}}^{x} \frac{P_{x}\left(x^{\prime}, N\right)}{\left[x^{\prime} \mid x_{0} ; N\right]} \frac{\Lambda\left(x^{\prime}, N\right)}{\nu} d x^{\prime}\right) .
$$

Take $\partial / \partial x$ of both sides, then use that $\frac{\partial}{\partial x}\left[x \mid x_{0} ; N\right]=-\left[x \mid x_{0} ; N\right] \frac{U(x, N)}{r}$ (see Eq. (3), where the dependences on $N$ are suppressed), and use Eq. (9) to substitute the expression in parentheses by $-P_{N}(x, N) /\left[x \mid x_{0} ; N\right]$ to obtain

$$
P_{x N}(x, N)+\frac{U(x, N)}{r} P_{N}(x, N)+\frac{\Lambda(x ; N)}{\nu} P_{x}(x, N)=0 .
$$


In addition, we have the initial condition $P(x, 0)=1, x \geq x_{0}$ (at $N=0$ the crack tip is at $\left.x_{0}\right)$ and the boundary condition

$$
P\left(x_{0}, N\right)=\exp \left(-\int_{0}^{N} \frac{\Lambda\left(x_{0}, N^{\prime}\right)}{\nu} d N^{\prime}\right), \quad N \geq 0
$$

(the probability that, by the cycle $N$, crack growth has not yet begun, i.e. no initiation at $x_{0}$ has occurred; c.f. Eq. (7)).

\section{Numerical Example, Paris Law}

In this section, we consider a tapered compact tension specimen (see Fig. 1) subjected to displacement controlled loading. If the displacement at the grips is $\delta$ and the specimen width is $a$, then $G(x)=[K(x)]^{2} / E$ (see, for example, [8]) where, for $z=x / a$,

$$
K(z)= \begin{cases}\frac{E \delta}{2 \sqrt{a}} \cdot \frac{(22.0 z+3.17) h^{3 / 2}(z)}{-115 h^{2}(z)+666 h(z)-483 \ln (3 h(z))-14.9}, & \text { if } 0 \leq z<0.42 \\ \frac{E \delta}{2 \sqrt{a}} \cdot \sqrt{-17.5 z^{3}+29.6 z^{2}-16.6 z+3.21}, & \text { if } 0.42 \leq z<0.52 \\ \frac{E \delta}{2 \sqrt{a}} \cdot \frac{(1.63 z+2.71) g^{3 / 2}(z)}{9.42 g^{2}(z)-14.1 g(z)+2.66 \ln (g(z))+17.1}, & \text { if } 0.52 \leq z<1.0\end{cases}
$$

where $h(z)=1 /(z+0.883), g(z)=1 /(1-z)$, and $E$ is the Young's modulus (see [7]). We also use

$$
\Lambda(x)=\left(\frac{G(x)}{2 \gamma^{*}}\right)^{0.2} .
$$

Cyclicity of the load is introduced by considering $\delta=\delta(N)=\delta_{0}(1+A \sin N)$. The illustrations below correspond to the parameter values that were used/determined in [7] (SI units): $\delta_{0}=0.35 \cdot 10^{-3}$, $a=40 \cdot 10^{-3}, x_{0}=6.5 \cdot 10^{-3}, E=3.1 \cdot 10^{9}, \gamma^{*}=24, \gamma_{\min }=0, \alpha=0.64$, $r=10^{-4}$. In addition, we use $\nu=1, A=0.5$.

\subsection{Evolution of Crack Depth Distribution}

Figure 2 shows evolution of crack depth distribution with the number of cycles. Plotted is the probability density $p(z, N)=\frac{\partial P(x, N)}{\partial(x / a)}=a p(x, N)$ for three different values of $N$.

The distributions owe their bi-modality to the non-monotonicity of the gradient of $G(x)$ for a tapered compact tension specimen (cf. Fig. 1). 


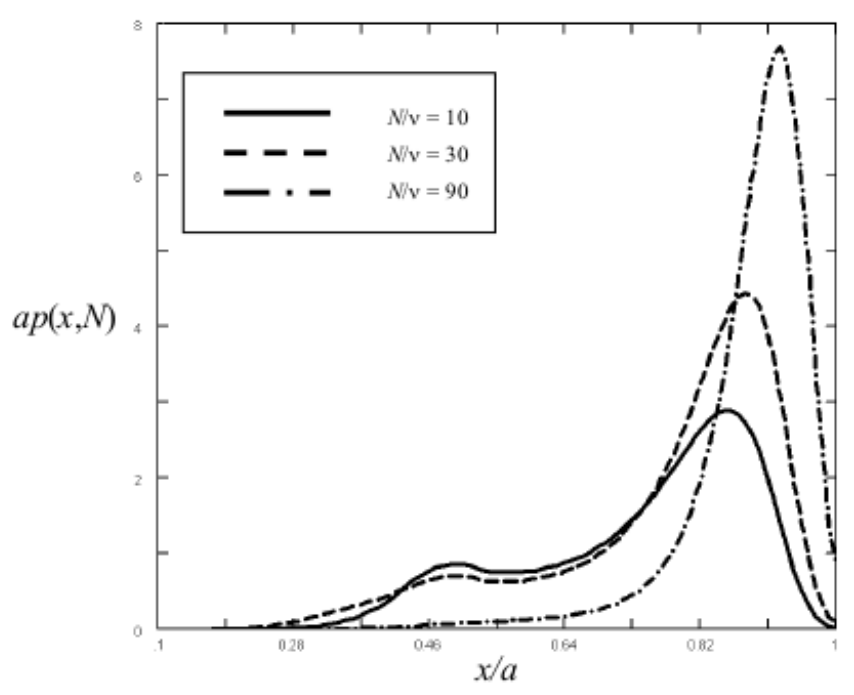

Figure 2: Probability densities of crack depth at various 'times'. Not shown is the atomic probability $P\left(x_{0}, N\right)$ at $x=x_{0}$, Eq. (11). The decrease of $P\left(x_{0}, N\right)$, as $N$ increases, accounts for the apparent increase of areas under the curves.

\subsection{Paris Law Type Relation}

In this sub-section, we examine the relation, predicted by the model, between the average crack speed and the amplitude of the stress intensity factor.

Notice that the notion of instantaneous crack speed, $V(x)$, is meaningless 'realization-wise'. Indeed, if one considers any realization of a (random) growing crack, then the speed of the crack tip takes one of two values, zero or infinity. Therefore, the average crack speed, $\langle V(x)\rangle$, cannot be defined as a straightforward average of $V(x)$.

Following [1], we define average crack propagation speed, $\hat{V}(x), x_{0}<x<a$, as follows. Let $\boldsymbol{N}(x)$ denote the (random) cycle at which the crack tip either arrives at or flies by $x, x_{0}<x<a$ and let $\langle\boldsymbol{N}(x)\rangle$ denote the average value of $\boldsymbol{N}(x)$. Evidently, the distribution function for $\boldsymbol{N}(x)$ is

$$
F_{\boldsymbol{N}(x)} N=\operatorname{Prob}\{\boldsymbol{N}(x) \leq N\}=\operatorname{Prob}\{\xi(N)>x\}=1-P(x, N)
$$


Therefore,

$$
\begin{aligned}
\langle\boldsymbol{N}(x)\rangle & =\int_{0}^{\infty} N d F_{N(x)}(N)=-\int_{0}^{\infty} N d P(x, N) \\
& =-\left[\left.N P(x, N)\right|_{0} ^{\infty}-\int_{0}^{\infty} P(x, N) d N\right] \\
& =\int_{0}^{\infty} P(x, N) d N .
\end{aligned}
$$

Finally, define

$$
\hat{V}(x)=\frac{1}{\frac{d}{d x}\langle N(x)\rangle} .
$$
useful:

Remark. In the case of constant load $(A=0)$ the following expression for $\frac{d\langle\boldsymbol{N}(x)\rangle}{d x}$ is

$$
\frac{d\langle\boldsymbol{N}(x)\rangle}{d x}=\int_{0}^{\infty} P_{x}(x, N) d N=-\int_{0}^{\infty} \frac{\nu}{\Lambda(x, N)}\left(P_{x N}(x, N)+\frac{U(x, N)}{r} P_{N}(x, N)\right) d N
$$

(one obtains it using Eq. (10)). Indeed, after dropping the dependence $\Lambda$ of and $U$ on $N$, factoring $\Lambda(x)$ outside of the integral, and rewriting the expression in the parentheses as

$$
\frac{\partial}{\partial N}\left(P_{x}(x, N)+\frac{U(x)}{r} P(x, N)\right)
$$

one gets $\frac{d\langle N(x)\rangle}{d x}=\frac{U(x)}{\Lambda(x)} \frac{\nu}{r}$ (to which corresponds $\left.\hat{V}(x)=\frac{\Lambda(x)}{U(x)} \frac{r}{\nu}\right)$.

Besides the crack speed $\hat{V}(x)$, Paris Law involves the "stress intensity factor", $K$. In our situation, $K=K(z)=K(z, \delta)$ is given by Eq. (12). For cyclic loading, let $K_{\max }$ and $K_{\min }$ denote the $K$-values that correspond to the highest and lowest values of the applied cyclic load, respectively (both $K_{\max }$ and $K_{\min }$ depend on the crack length). Denote $\Delta K=K_{\max }-K_{\min }$. Evidently, $\Delta K=\Delta K(z)=K\left(z, \delta_{0}\right) \cdot 2 A$.

Paris Law summarizes numerous observations in fatigue crack growth, namely, that crack speed $V$ is proportional to a power of the amplitude $\Delta K$ of the stress intensity factor over a wide range of the values of $\Delta K$. It is common to refer to the $\hat{V}$-vs.- $\Delta K$ relation as 'linear in log-log scale'. Also it is worth noting that the commonly observed dependence of $\ln (\hat{V})$ on $\ln (\Delta K)$ is steeper outside of the (approximately) linear range.

In Fig. 3, we show the relation between $\ln \hat{V}$ and $\ln (\Delta K)$ predicted by the model (after finding $\hat{V}=\hat{V}(z)$ and excluding $z$ from $\hat{V}(z)$ and $\Delta K(z)$ ).

The 'kink' in the lower part of the curve has the following origin. The curve is parametrized by $z$. As $z$ increases from $x_{0} / a$ to $1, K(z)$ decreases and so the point on the curve travels from the upper right corner down. The $K$ values 


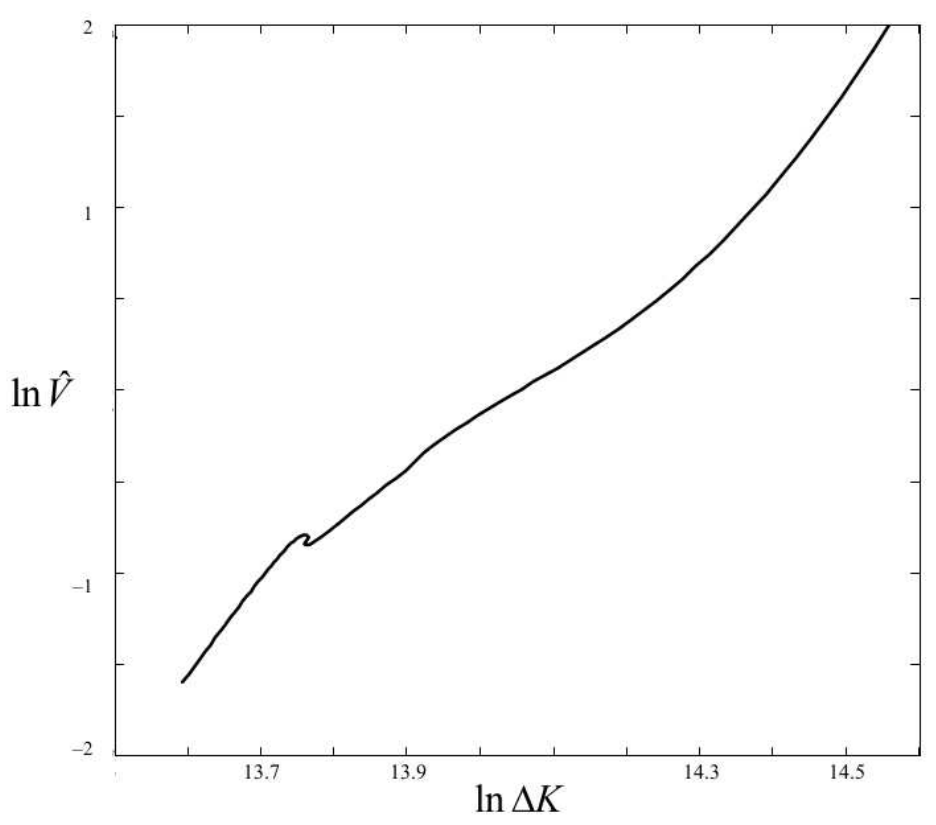

Figure 3: Dependence of $\ln (\hat{V})$ on $\ln (\Delta K)$ predicted by the model.

around the kink correspond to the range of $z$ values $(0.47 \lesssim z \lesssim 0.62)$, for which $K$ stays approximately constant (cf. Fig. 1 where essentially the square of $K(z)$ is plotted). It is worth noting that Fig. 3 is qualitatively similar to a typically observed relationship between the crack speed and the amplitude of the stress intensity factor.

\section{References}

[1] B. Kunin, I. Savin, Application of Double-Poisson Process in Brittle Fracture, International Journal of Pure and Applied Mathematics, 72, No. 4 (2011), 501-514.

[2] M. Ostoja-Starzewski, G. Wang, Particle Modeling of Random Crack Patterns in Epoxy Plates, Probabilistic Engineering Mechanics, 21, No. 3 (2006), 267-275. 
[3] A. Chudnovsky, B. Kunin, On applications of probability in fracture mechanics, in Computational Mechanics of Probabilistic and Reliability Analysis, (ed's W.K. Liu and T. Belytschko), Elmepress International, Washington (1989), 395-415.

[4] A. Chudnovsky, B. Kunin, A probabilistic model of brittle crack formation, J Appl Phys, 62, No. 10, (1987), 4124-4129.

[5] A. Chudnovsky, B. Kunin, M. Gorelik, Modeling of brittle fracture based on the concept of crack trajectory ensemble, Engineering Fracture Mechanics, 58, No's. 5-6, (1997), 437-457.

[6] M. A. I. Mul, A. Chudnovsky, A. Moet, A probabilistic approach to fracture toughness of composites, Philosophical Magazine A 56, No. 3 (1987), 419443.

[7] B. Kunin, R. S. Dearth, Evaluation of statistical fracture toughness parameters on the basis of crack arrest experiment, Probabilistic Engineering Mechanics, 13, No. 3 (1998), 139-146.

[8] M. Isida, Arbitrary loading problems of doubly symmetric regions containing a central crack, Engineering Fracture Mechanics, 7, (1975), 505-514. 